\title{
Investigation of Preeclampsia Using Raman Spectroscopy
}

\author{
Günay Başar, ${ }^{1}$ Uğur Parlatan, ${ }^{1,2}$ Şeyma Şeninak, ${ }^{3}$ \\ Tuba Günel, ${ }^{4}$ Ali Benian, ${ }^{5}$ and İbrahim Kalelioğlu ${ }^{6}$ \\ ${ }^{1}$ Department of Physics Engineering, Faculty of Science and Letters, Istanbul Technical University, \\ Maslak, 34469 Istanbul, Turkey \\ ${ }^{2}$ Department of Engineering Science, Faculty of Engineering, Istanbul University, \\ Avcilar, 34320 Istanbul, Turkey \\ ${ }^{3}$ Physics Department, Faculty of Science, Istanbul University, Vezneciler, 34134 Istanbul, Turkey \\ ${ }^{4}$ Department of Molecular Biology and Genetics, Faculty of Science, Istanbul University, \\ Vezneciler, 34134 Istanbul, Turkey \\ ${ }^{5}$ Department of Obstetrics and Gynecology, Cerrahpasa Medical Faculty, Istanbul University, \\ Fatih, 34098 Istanbul, Turkey \\ ${ }^{6}$ Department of Obstetrics and Gynecology, Istanbul Medical Faculty, Istanbul University, \\ Capa, 34093 Istanbul, Turkey
}

Correspondence should be addressed to Günay Başar, basar@itu.edu.tr

Copyright (C) 2012 Günay Başar et al. This is an open access article distributed under the Creative Commons Attribution License, which permits unrestricted use, distribution, and reproduction in any medium, provided the original work is properly cited.

\begin{abstract}
Preeclampsia is associated with increased perinatal morbidity and mortality. There have been numerous efforts to determine preeclampsia biomarkers by means of biophysical, biochemical, and spectroscopic methods. In this study, the preeclampsia and control groups were compared via band component analysis and multivariate analysis using Raman spectroscopy as an alternative technique. The Raman spectra of serum samples were taken from nine preeclamptic, ten healthy pregnant women. The Band component analysis and principal component analysis-linear discriminant analysis were applied to all spectra after a sensitive preprocess step. Using linear discriminant analysis, it was found that Raman spectroscopy has a sensitivity of $78 \%$ and a specificity of $90 \%$ for the diagnosis of preeclampsia. Via the band component analysis, a significant difference in the spectra of preeclamptic patients was observed when compared to the control group. 19 Raman bands exhibited significant differences in intensity, while 11 of them decreased and eight of them increased. This difference seen in vibrational bands may be used in further studies to clarify the pathophysiology of preeclampsia.
\end{abstract}

Keywords: Raman spectroscopy, Preeclampsia, Multivariate analysis, Band component analysis

\section{Introduction}

Preeclampsia is a pregnancy-specific disorder that affects up to $15 \%$ of all pregnancies. The specific conditions of the disease can basically be defined as hypertension $(\geq 140 / 90 \mathrm{mmHg})$ and proteinuria. These conditions occur after the 20-22th week of pregnancy [1]. Preeclampsia brings risks to both mother and baby and causes maternal morbidity and fetal mortality [2]. 
One of the causes of preeclampsia is thought to be a lack of antioxidants [3]. Free radicals or reactive oxygen species can interact with lipids and proteins which may cause lipid peroxidation [4] and protein modification [5]. It is suggested that the alteration of lipid and protein levels is correlated with oxidative stress and vascular dysfunction in preeclampsia [4].

The aetiology and pathology of preeclampsia are still of great interest to researchers. There have been numerous efforts to determine preeclampsia biomarkers employing biophysical, biochemical, and spectroscopic methods. D'Aniello et al. [6] used a high-performance liquid chromatography (HPLC) fluorometric method to predict the preeclampsia serum biomarkers and reported reduced levels of free amino acids, especially arginine levels which dramatically decreased in preeclampsia patients. On the other hand, Glew et al. [7] found no significant difference between the amino acid levels of preeclamptic and control groups' plasma samples. On the contrary, López-Quesada [8] reported an increase in most amino acid levels. Turner et al. [9] obtained higher tyrosine, histidine, and phenylalanine concentrations for preeclamptic plasma samples using nuclear magnetic resonance spectroscopy. In another study [10], they reported lower lipid concentrations for preeclamptic plasma samples. On the contrary, there are studies reporting elevated lipid levels in the serum of preeclamptic patients. Robinson et al. [11] showed altered lipid metabolism characterized by elevated circulating triglycerides and nonesterified free fatty acids in preeclampsia. Hubel et al. [12] found a decreased particle size of low-density lipoprotein and increased triglyceride, free fatty acids, total cholesterol, and LDL-cholesterol concentrations in preeclampsia.

A very recent study which used Fourier transform infrared spectroscopy [13] reported decreased intensities for protein bands from the spectra of lyophilized serum samples. Furthermore, they found a relationship between preeclampsia and lipid parameters, such as increased triglycerides and decreased HDL-cholesterol during preeclampsia.

A study of the available literature reveals conflicting views about determining the serum levels of these biomarkers in body fluids from healthy and preeclamptic women. Therefore, there is a need for complementary studies to explore the pathophysiology of preeclampsia.

Raman spectroscopy is a potent tool that gives information about molecular structures. Raman spectroscopy is based on vibrational transitions between one vibrational state and a virtual state as a result of the inelastic collision of light and molecules. The frequencies of transition lines and their intensities are characteristic for each molecular vibration which may provide information about the conformation of macromolecules such as proteins [14, 15], nucleic acids [16, 17], and lipids [18, 19]. Thus Raman spectroscopy can be a suitable tool to determine biomarkers which may play a role in the pathophysiological mechanism of diseases. Several diseases were investigated on human body fluids such as whole blood [20, 21] and blood serum [22, 23] by using Raman spectroscopy.

Multivariate methods with Raman spectroscopy in the determination of the concentration of some constituents in serum or whole blood were performed. The partial least squares method [24-28] was used to predict the concentration of blood analytes including glucose, urea, cholesterol, triglycerides, total protein, albumin, and hemoglobin. Furthermore, a number of studies have elucidated the diagnostic potential of Raman spectroscopy combined with multivariate analysis techniques for several diseases [29-34].

To our knowledge, this is the first Raman spectroscopic study on preeclampsia to exist in the literature. In the study, we measured the Raman spectra of serum samples taken from preeclamptic and healthy women. We analyzed the measured spectra with band component analysis and applied principal 
component analysis-linear discriminant Analysis (PCA-LDA) after a sensitive preprocess step to find the differences between preeclamptic and healthy women.

\section{Materials and Methods}

\subsection{Sample Collection and Preparation}

Informed consent for the study was obtained from all the women concerned, and approval was given by the Ethics Committee of the Faculty of Medicine, Istanbul University. The sample population consisted of ten normotensive women who had no underlying medical conditions and nine otherwise healthy preeclamptic women who were diagnosed at Cerrahpasa Medical Faculty, Istanbul University. The normal pregnancies were followed until their completion, and no preeclampsia occurred.

Blood samples taken in $10 \mathrm{~mL}$ gel tubes were centrifuged during a 10-minute period with $3000 \mathrm{rpm}$ to obtain serum samples. Each serum sample was prepared in two aliquots which were measured on two different days to validate the stability of the experimental conditions. The samples were kept at $-20^{\circ} \mathrm{C}$ until the observation day and were measured maximum in seven days. Frozen serum samples in eppendorf tubes were defrosted by hand warmth before the Raman measurements were taken. About $1 \mathrm{~mL}$ of serum samples were prepared in glass cuvettes which were cleaned properly after every measurement.

\subsection{Experiment}

The experimental setup consists of a $785 \mathrm{~nm}, 100 \mathrm{~mW}$ single mode diode laser (CrystaLaser) and a spectrograph ( $f=303 \mathrm{~mm}, f \# 4.3$, Andor) equipped with a 600 lines $/ \mathrm{mm}$ grating and with TE cooled CCD $\left(-90^{\circ} \mathrm{C}\right.$, Andor iDus DU420A-OE). A Faraday isolator (EOTech, BB8-00011) was placed just in front of the diode laser to prevent back reflections which cause instabilities on single longitudinal mode operation and the power of the laser. A laser line filter was used to obtain a clear laser emission (Semrock, LL01-780-12.5). The laser beam was directed and then focused on the sample by suitable mirrors and lenses. Back scattered light from the sample was collected at $180^{\circ}$ geometry by the same lens. The scattered light from the sample was filtered two times successively with Raman edge filters which transmit only the Raman scattering (inelastic scattering) beam while they reflect the Rayleigh (elastic scattering) beam. The Raman scattering beam was inserted into the spectrograph through $100 \mu \mathrm{m}$ entrance slit by an achromat lens with a focal length of $50 \mathrm{~mm}$.

The Raman spectra of 38 serum samples (nine preeclamptic and ten healthy; two from each patient) were taken with a $30 \mathrm{~s}$ exposure time, 20 successive scans over a 10-minute period. The laser power on the sample was about $50 \mathrm{~mW}$. After cosmic-ray removal, the 20 scans were averaged for each sample. The wavenumber calibration of the spectrograph was performed with reference bands of toluene [35]. A toluene Raman spectrum was taken for one second after each Raman spectrum of serum sample to apply a developed iterative calibration technique which minimizes the effects of possible spectral shifts during the measurement day(s). These shifts may arise from some reasons such as temperature, pressure, humidity which may affect the diode laser, spectrograph, and all optical components. The mean Raman spectrum of all serum samples for control and preeclamptic groups and the Raman spectrum of water are shown in Figure 1(a). The Raman spectrum of water was taken at the end of each measurement 


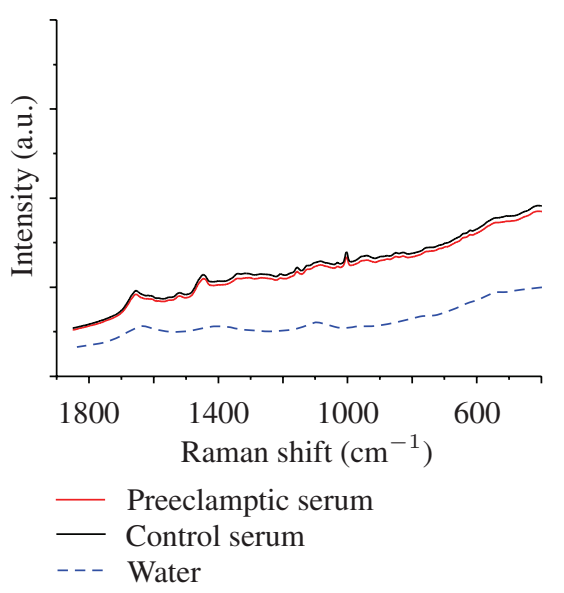

(a)

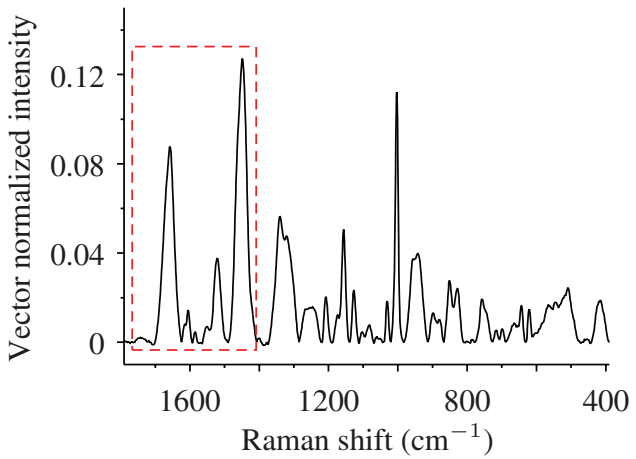

(b)

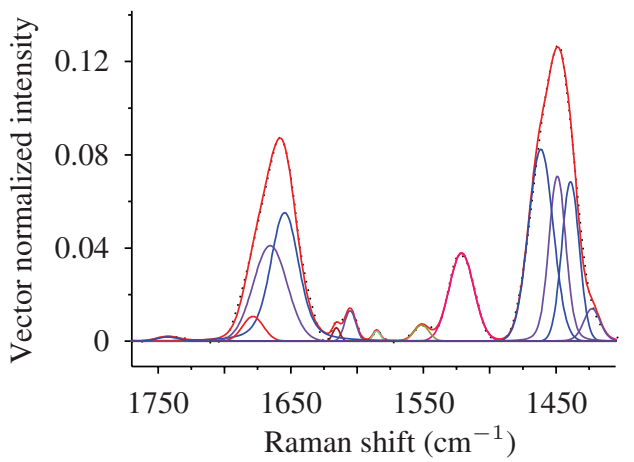

(c)

Figure 1: (a) The raw mean Raman spectra of all serum samples for healthy and preeclamptic groups and the Raman spectrum of water, (b) Raman spectrum of a serum sample taken from a healthy pregnant after water background is subtracted, baseline corrected, and vector normalized and (c) Raman spectrum (dotted) of a spectral part of (b) (marked with dotted rectangle) with fit curve (solid line) and 13 band components (other colored lines).

day. The wide Raman band from water at around $1640 \mathrm{~cm}^{-1}$ and other wide bands from glass at around $1400 \mathrm{~cm}^{-1}$ and $1100 \mathrm{~cm}^{-1}$ are visible. However, the background correction by subtracting the water Raman spectrum from each serum spectrum eliminates these interferences.

Although the fluorescence could be significantly reduced with the excitation at $785 \mathrm{~nm}$, the residual fluorescence which originates from macromolecules in serum [36], from glass and also stray light contributions, should be eliminated. Therefore, baseline corrections were applied by fitting a cubic spline curve for each spectrum and subtracting the curves from the spectra. The wavenumbers corresponding to the data points that constitute the curve for the baseline were chosen to be identical for each curve in order to assure objectivity among the processed spectra. Then all spectra were vector normalized. The baseline corrected, vector normalized mean Raman spectrum of control samples is given in Figure 1(b). 


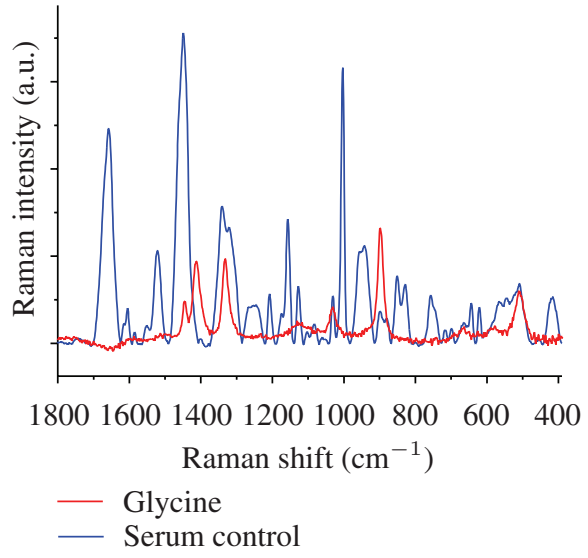

(a)

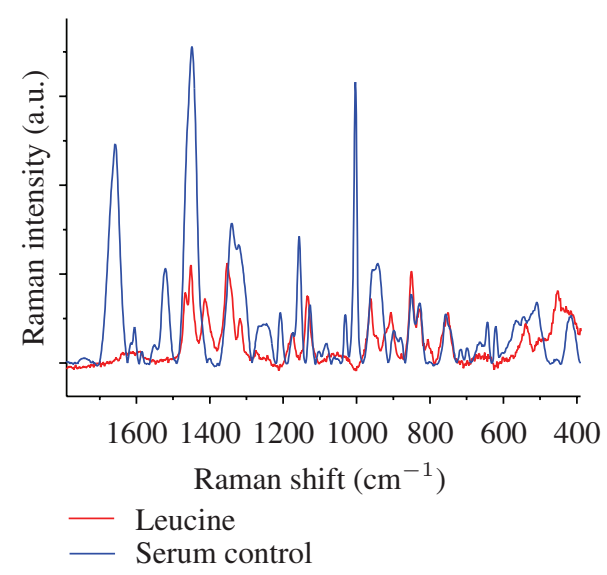

(b)

Figure 2: Raman spectra of $0.1 \mathrm{M}$ glycin (a) and leucine (b) in water are presented with mean Raman spectrum of control samples.

Second derivative spectra were used to determine the number and initial positions of the components under broad bands. By using this information all spectra were fitted with a Voigt profile using commercial software, Grams/AI 8.0, to determine band intensities and positions. Figure 1(c) is an example of a fit of the part of the serum Raman spectrum given in Figure 1(b).

Additionally, the Raman spectra of 15 amino acids, $\beta$-carotene, glucose, glutathione, adenine, urea, and serum albumin in water $(0.1 \mathrm{M})$ were measured individually to determine the band positions and intensities. As an example, the Raman spectra of $0.1 \mathrm{M}$ glycine and L-leucine in water are given in Figure 2 superposed with the mean Raman spectrum of serum samples of the control group to show the contribution of glycine and leucine bands. All of the chemicals used in this work were purchased from Merck and Sigma.

\subsection{Statistical Analysis}

All of the measured Raman spectra established a data set for PCA and LDA which are two of the most widely used data classification methods. In the PCA process, all spectra constitute a matrix whose rows are observations and columns are wavenumbers. The PCA software first applies a mean centering on this matrix and then determines the eigenvalues and eigenvectors of the covariance matrix of the data and thus maximizes the variance between variables. Therefore, defining eigenvector $V$, transpose of eigenvector $V^{\prime}$, scores $T$ and mean spectrum $A$; PCA score vectors are expressed as below:

$$
T=V^{\prime} A \text {. }
$$


Having defined original spectrum $X$, the expression below can be obtained by means of scores and loadings:

$$
X=A+T V+\varepsilon
$$

where $\varepsilon$ means residual matrix [48, 49]. In spectral analysis, the scores lead to a classification between groups. Since eigenvalues are sorted in descending order in the PCA process, when the first most important scores are plotted versus each other, spectral differences will occur as discriminations in score plot.

The LDA analysis was applied using Fisher's criterion which is actually a Bayesian decision rule. Defining

$$
\begin{gathered}
w=\Sigma^{-1}\left(\mu_{1}-\mu_{2}\right), \\
w_{0}=-\frac{1}{2}\left(\mu_{1}+\mu_{2}\right) \Sigma^{-1}\left(\mu_{1}-\mu_{2}\right)^{T}-\log \left(\frac{\pi_{1}}{\pi_{2}}\right),
\end{gathered}
$$

Fisher's criterion is given by

$$
w^{T} x+w_{0}>0
$$

where $\mu_{1}$ and $\mu_{2}$ are group mean vectors, $\pi_{1}$ and $\pi_{2}$ are prior probabilities for each group, $\mathbf{x}$ is a row vector which consists of two most important PC scores, and $\boldsymbol{\Sigma}$ is pooled covariance matrix [48, 50].

PCA and LDA processes were applied to all preprocessed spectra. All PCA analyses were made on Grams PLS Plus/IQ software with multiplicative scatter correction preprocessing and leave-one-out cross-validation.

The LDA process was performed with software programmed on MATLAB 7.4.0.

\section{Results}

The preeclamptic and normotensive groups were well matched. There was no significant difference for age, parity, and gestational age at blood sampling between the groups $(P>0.05)$. The Raman spectra of 38 serum samples (18 preeclamptic and 20 healthy) were obtained. The vector normalized mean spectra of serum samples of the preeclamptic and control groups are shown in Figure 3 . The figure demonstrates that there are differences in some band intensities between the two spectra indicated with arrows and corresponding wavenumbers. The differences of some Raman bands between normalized mean spectra in Figure 3 are not visible since many Raman bands consist of multiple band components (see Figure 1(c)). However, the changes in band intensities can better be seen in the difference spectrum.

All spectra were fitted with a Voigt profile as mentioned previously. The vibrational band intensities and positions in wavenumbers were determined for the control and preeclamptic groups according to fit results. The vibrational bands exhibiting differences in intensities $(P<0.05)$ are given in Table 1 with tentative band assignments. Additionally, the possible contributions of the constituents to 


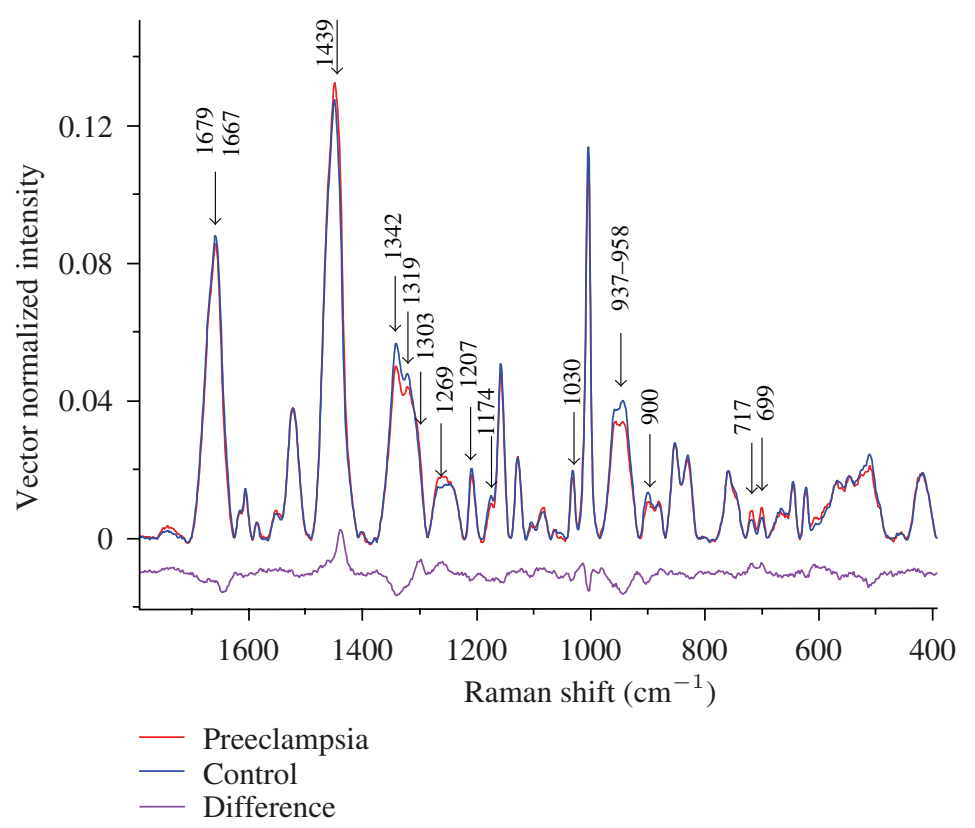

Figure 3: Normalized mean Raman spectra of serum samples taken from control and preeclamptic group. The lowest spectrum is the difference spectrum (shifted down for clarity).

vibrational bands on the Raman spectra of serum were determined with the measurements of the Raman spectra of amino acids and other ingredients mentioned above. Their contributions to the vibrational bands were also represented in Table 1.

The vibrational bands in Table 1 were divided into two groups according to alterations in band intensities. As can be seen from the table, the intensities of 11 Raman bands decreased and eight Raman bands increased in preeclamptic samples when compared to the control group. The seven Raman bands with reduced band intensities are all protein bands presented above with possible main contributions from amino acids glycine, glutamine, valine, leucine, phenylalanine, tyrosine, and histidine. Other significant decreases in band intensities for preeclamptic samples are observed at the bands at 958, 1342,1448 , and $1679 \mathrm{~cm}^{-1}$. Regarding these bands, 958 and $1448 \mathrm{~cm}^{-1}$ have contributions from both amino acids and lipids. The band at $1342 \mathrm{~cm}^{-1}$ can be ascribed to $\mathrm{CH}$ bending of many amino acids, and the band at $1679 \mathrm{~cm}^{-1}$ can be assigned to one of the coupled modes of the polypeptide backbone, the so-called amid I band. Lipids contribute to all of the bands of increasing intensities and amino acids also contribute to most of these bands.

A PCA was performed by using five factors which were determined by selecting an eigenvalue number that gives minimum slope of the scree plot and by taking into account the total variance explained values under the threshold after testing the accuracy of the analysis by leave-one-out crossvalidation. PCA was applied to all spectra which build up a matrix as an input. This gives scores and loadings as outputs. The scatter plot of the scores PC2 versus PC3 and spectra of their loadings in spectral range of $600 \mathrm{~cm}^{-1}-1760 \mathrm{~cm}^{-1}$ with the assigned Raman bands (given in Table 1) are shown together in Figure 4. Among the first five most important component scores, PC 2 and PC 3 were found 
Table 1: Changing vibrational relative band intensities in serum samples from two groups are given with tentative band assignment. Significance values of band intensities were calculated using Mann-Whitney $U$ analysis with $95 \%$ confidence level.

\begin{tabular}{|c|c|c|c|c|c|}
\hline \multirow{2}{*}{$\begin{array}{l}\text { Wavenumber } \\
\left(\mathrm{cm}^{-1}\right)\end{array}$} & \multicolumn{2}{|c|}{ Normalized band intensities (a. u.) } & \multirow{2}{*}{$\begin{array}{c}\text { Tentative band } \\
\text { assignment } \\
{[37-47]}\end{array}$} & \multirow{2}{*}{ Contributions } & \multirow{2}{*}{$P$} \\
\hline & Preeclampsia & Control & & & \\
\hline \multicolumn{6}{|c|}{ Decreased band intensities in preeclampsia } \\
\hline 900 & $0.009 \pm 0.002$ & $0.012 \pm 0.001$ & $\nu(\mathrm{C}-\mathrm{C})$ & $\begin{array}{c}\text { Glycine, } \\
\text { glutathione, } \\
\text { glutamine }\end{array}$ & $<0.001$ \\
\hline 937 & $0.026 \pm 0.004$ & $0.030 \pm 0.001$ & $\nu\left(\mathrm{C}_{\alpha}-\mathrm{C}\right)$ & Valine & $<0.001$ \\
\hline 948 & $0.021 \pm 0.003$ & $0.023 \pm 0.001$ & $\nu(\mathrm{C}-\mathrm{C})$ & Valine & 0.014 \\
\hline 958 & $0.025 \pm 0.004$ & $0.030 \pm 0.002$ & $\nu(\mathrm{C}-\mathrm{C})$ & Leucine, lipids & $<0.001$ \\
\hline 1030 & $0.017 \pm 0.004$ & $0.020 \pm 0.003$ & $\delta(\mathrm{C}-\mathrm{H})$ & $\begin{array}{l}\text { Phe, } \\
\text { glutathione }\end{array}$ & 0.021 \\
\hline 1174 & $0.010 \pm 0.002$ & $0.012 \pm 0.001$ & $\begin{array}{c}\mathrm{NH}_{3} \text { rock, } \\
\delta(\mathrm{C}-\mathrm{H})\end{array}$ & Tyr, leucine & $<0.001$ \\
\hline 1207 & $0.019 \pm 0.003$ & $0.021 \pm 0.003$ & $\nu\left(\mathrm{C}-\mathrm{C}_{6} \mathrm{H}_{5}\right)$ & Phe, Tyr & 0.023 \\
\hline 1319 & $0.042 \pm 0.004$ & $0.045 \pm 0.003$ & $\begin{array}{c}\mathrm{CH}_{3} \mathrm{CH}_{2} \\
\text { twisting }\end{array}$ & $\begin{array}{c}\text { Histidine, } \\
\text { valine, leucine }\end{array}$ & 0.015 \\
\hline 1342 & $0.044 \pm 0.005$ & $0.051 \pm 0.003$ & $\delta(\mathrm{CH})$ & $\begin{array}{l}\text { Some amino } \\
\text { acids }\end{array}$ & $<0.001$ \\
\hline 1448 & $0.062 \pm 0.012$ & $0.073 \pm 0.006$ & $\begin{array}{l}\delta\left(\mathrm{CH}_{2}\right), \\
\delta\left(\mathrm{CH}_{3}\right)\end{array}$ & $\begin{array}{l}\text { Some amino } \\
\text { acids, lipids }\end{array}$ & 0.009 \\
\hline 1679 & $0.012 \pm 0.003$ & $0.015 \pm 0.002$ & Amide I & Protein & 0.001 \\
\hline \multicolumn{6}{|c|}{ Increased band intensities in preeclampsia } \\
\hline 699 & $0.009 \pm 0.002$ & $0.006 \pm 0.001$ & $\nu(\mathrm{C}-\mathrm{S})$ & $\begin{array}{l}\text { Lipids, } \\
\text { methionine }\end{array}$ & $<0.001$ \\
\hline 717 & $0.008 \pm 0.002$ & $0.006 \pm 0.001$ & $\begin{array}{l}\mathrm{N}^{+}\left(\mathrm{CH}_{3}\right)_{3} \\
(=\mathrm{C}-\mathrm{H}) \text { in }\end{array}$ & Lipids & $<0.001$ \\
\hline 1269 & $0.10 \pm 0.04$ & $0.07 \pm 0.03$ & $\begin{array}{l}\text { plane bending, } \\
\text { amide III }\end{array}$ & Protein, lipids & 0.001 \\
\hline 1303 & $0.027 \pm 0.004$ & $0.024 \pm 0.002$ & $\begin{array}{l}\delta\left(\mathrm{CH}_{2}\right) \\
\text { twisting }\end{array}$ & Lipids & 0.014 \\
\hline 1427 & $0.022 \pm 0.003$ & $0.018 \pm 0.002$ & $\mathrm{CH}_{2}$ def. & $\begin{array}{l}\text { Some amino } \\
\text { acids, lipids }\end{array}$ & $<0.001$ \\
\hline 1439 & $0.071 \pm 0.008$ & $0.058 \pm 0.004$ & $\mathrm{CH}_{2}$ def. & $\begin{array}{l}\text { Some amino } \\
\text { acids, lipids }\end{array}$ & $<0.001$ \\
\hline 1460 & $0.095 \pm 0.004$ & $0.089 \pm 0.002$ & $\mathrm{CH}_{2}, \mathrm{CH}_{3}$ def. & $\begin{array}{l}\text { Some amino } \\
\text { acids, lipids }\end{array}$ & $<0.001$ \\
\hline 1667 & $0.041 \pm 0.002$ & $0.035 \pm 0.003$ & $\begin{array}{c}\nu(\mathrm{C}=\mathrm{C}), \\
\text { amide I }\end{array}$ & Protein, lipids & $<0.001$ \\
\hline
\end{tabular}

$\nu$ : stretching mode; $\delta$ : bending mode; def.: deformation. 


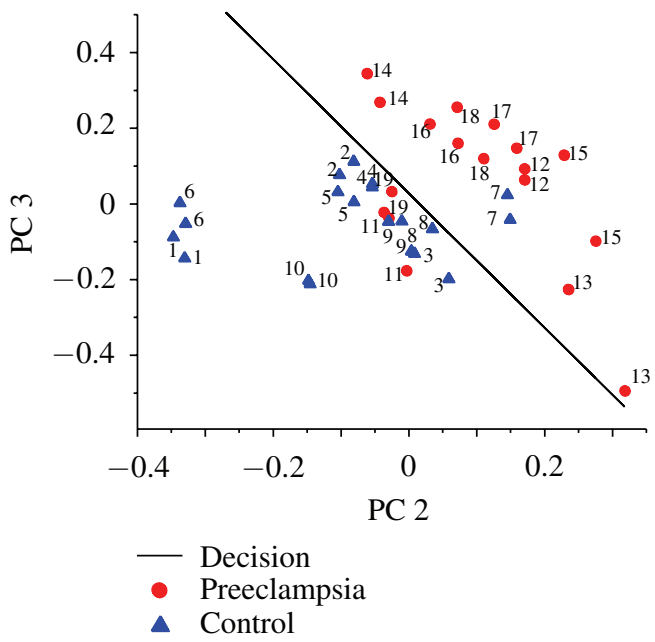

(a)

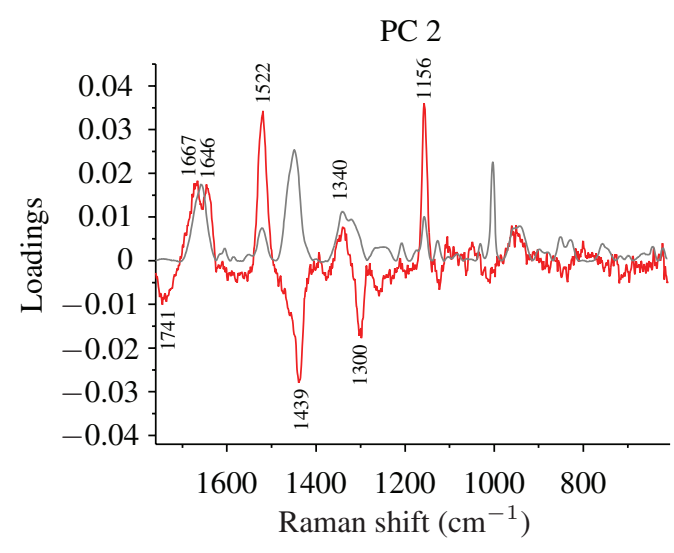

(b)

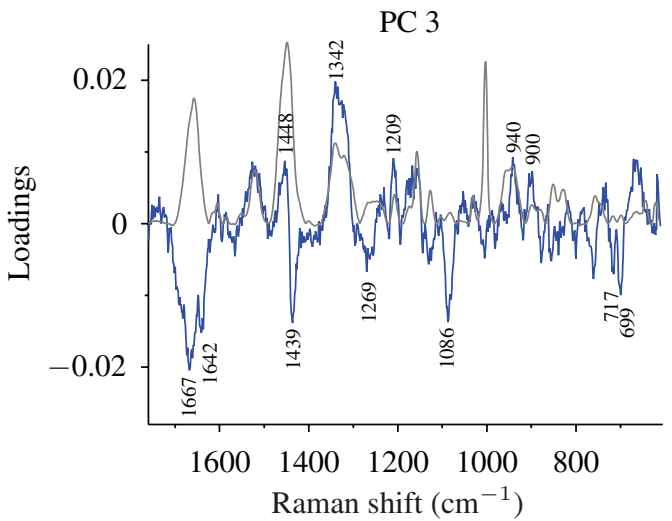

(c)

Figure 4: (a) The scatter plot of the scores PC 2 versus PC 3, corresponding loadings (b) PC 2 and (c) PC 3. The numbers in (a) represent control (1-10) and preeclamptic (11-19) samples.

to be significant in the $99 \%$ confidence level. Therefore, PC 2 and PC 3 loadings were used to provide chemical information.

To determine a quantitative discrimination, we applied our LDA code on the first two most important PCA scores. Fisher's criterion was used to obtain a decision line to discriminate two groups from each other which is superposed in Figure 4(a). The graph shows that the two groups are clearly separated from each other. The duplicate numbers indicate the samples (1-10 control and 1119 preeclampsia) taken from the same person measured on different days as mentioned previously. The decision line demonstrates that two healthy samples from one person and four diseased samples from two patients were misclassified. The results of PCA-LDA analysis applied to all serum samples summarized in Table 2 with $78 \%$ sensitivity and $90 \%$ specificity. 
Table 2: Summary of PCA-LDA results for serum samples.

\begin{tabular}{lcc}
\hline Raman measurement & Preeclamptic & Healthy \\
\hline+ & 14 & 18 \\
- & 4 & 2 \\
\hline
\end{tabular}

PC 2 and PC 3 loadings are presented in Figures 4(b) and 4(c) superposed with control mean Raman spectrum. Two sharp Raman bands at $1156 \mathrm{~cm}^{-1}$ and at $1522 \mathrm{~cm}^{-1}$ belong to betacarotene Raman bands appear in Figure 4(b) since they scattered strongly in intensity especially for the preeclamptic patients. They are absent in Table 1 because these bands showed a slight change in average intensity according to the band component analysis. Moreover, their intensities were also found to be insignificant $(P>0.05)$ according to the Mann-Whitney $U$ test. PC 2 loading mainly shows negative variations for lipid bands which are at 1300,1439 , and $1741 \mathrm{~cm}^{-1}$, while $\mathrm{CH}$ bending mode at 1340 and amide I band at around $1650 \mathrm{~cm}^{-1}$ exhibit positive variation. Although PC 3 shows more noisy contributions than PC 2, it exhibits the same trend like in PC 2 for protein and lipid bands except amide I band given in Figure 4(c). Protein bands at 1642, 1667 (amide I), and $699 \mathrm{~cm}^{-1}$ show negative variations, and the ones at $900,940,1209,1342$, and $1448 \mathrm{~cm}^{-1}$ show positive variations. On the other hand, lipid bands at 717, 1086, 1269, and $1439 \mathrm{~cm}^{-1}$ exhibit negative variation. Some of these bands do not appear in Table 1 since the significance values of those bands' intensities are higher than 0.05 .

\section{Discussion}

By means of band component analysis, it is observable that the intensities of 11 Raman bands decreased and eight Raman bands increased in the preeclamptic samples. Although the decreasing bands in intensities are probably related to the bands of the individual amino acids (e.g., Figure 2) given in Table 1, it is difficult to conclude that a certain amino acid is responsible for the corresponding decrease because of the complexity of the spectrum. However, protein-related bands in the spectra tend to decrease in intensity for preeclamptic patients. It is well known that proteins are vulnerable to the attack from free radicals and related oxidants $[4,51]$. When attacked by free radicals, amino acids are converted to their oxidative derivatives [52] which may be the reason for the decrease in the protein band intensities.

A significant increase in the vibrational band intensities of preeclamptic samples was found at $699,717,1269,1303,1427,1439,1460$, and $1667 \mathrm{~cm}^{-1}$ which are all related to lipids as given in Table 1.

In some studies, [11-13] elevated lipid concentrations are found in preeclampsia, which are also consistent with our results. Increased lipid concentrations are known to be a major risk factor for cardiovascular diseases which is a known cause of endothelial dysfunction also seen in preeclampsia [1].

It can be seen from Table 1 that although the differences in increasing or decreasing band intensities are almost within the standard deviations, they exhibit significant differences in the intensities between the two groups $(P<0.05)$. However, many of the bands are formed by multiple amino acids, lipids, or other constituents in the serum. Therefore, the individual contributions of amino acids to these 
differences in band intensities remain unknown. Moreover, some weak bands in Table 1 may not be considered as reliable components of spectra since their intensities are almost at the level of noise.

The use of Raman spectroscopy combined with multivariate statistical methods gives rise to the classification between samples and helps to determine which variables change in disease states when compared to healthy samples. The linear discriminant analysis calculation after PCA exhibits a clear separation between the preeclamptic and control groups which can be seen in Figure 4(a).

It is to be remarked that each pair of data points belonging to the same sample is closely located in the graph. Although the pairs were measured on different days, they gave similar results. This means that systematic errors due to experimental conditions do not have any noticeable effect on the results.

We predicted preeclampsia with $78 \%$ specificity and $90 \%$ sensitivity by using multivariate analysis with Raman spectroscopy. The results obtained may be interpreted as low for the diagnosis of a disease.

PC 2 and PC 3 loadings spectra are consistent with the results of band component analysis.

These results may be improved by applying further multivariate techniques with larger numbers of samples. Nevertheless, Raman spectroscopy may provide a noninvasive method for studies exploring the pathophysiology of preeclampsia.

\section{Conclusion}

This study is the first Raman spectroscopic study on preeclampsia. When the control and preeclamptic groups were compared, alterations at some Raman bands of serum samples were observed. The contributions of some biomarkers that may lead to these changes were determined by using Raman spectroscopic measurements. These alterations on lipid bands and also on protein bands assigned to main contributions from particular amino acids may be investigated in further studies in respect of lipid bands and protein bands that focus on explaining the pathophysiology of the disease. They may also be tested in studies trying to predict the existence of preeclampsia. Although studies that use more samples may be required in order to confirm our results, our preliminary findings may offer a pioneering basis for further studies.

\section{Acknowledgment}

S. Seninak and U. Parlatan are grateful to the support offered by the research fund of Istanbul University, Project no. 4328 and 4330, respectively.

\section{References}

[1] J. M. Roberts, "Preeclampsia: what we know and what we do not know," Seminars in Perinatology, vol. 24, no. 1, pp. 24-28, 2000.

[2] C. A. Hubel, "Oxidative stress in the pathogenesis of preeclampsia," Proceedings of the Society for Experimental Biology and Medicine, vol. 222, no. 3, pp. 222-235, 1999.

[3] J. B. Sharma, A. Sharma, A. Bahadur, N. Vimala, A. Satyam, and S. Mittal, "Oxidative stress markers and antioxidant levels in normal pregnancy and pre-eclampsia," International Journal of Gynecology and Obstetrics, vol. 94, no. 1, pp. 23-27, 2006. 
[4] U. Adiga, V. D’Souza, A. Kamath, and N. Mangalore, "Antioxidant activity and lipid peroxidation in preeclampsia," Journal of the Chinese Medical Association, vol. 70, no. 10, pp. 435-438, 2007.

[5] K. J. Davies, "Protein damage and degradation by oxygen radicals. I. general aspects," Journal of Biological Chemistry, vol. 262, no. 20, pp. 9895-9901, 1987.

[6] G. D'Aniello, A. Tolino, and G. Fisher, "Plasma L-arginine is markedly reduced in pregnant women affected by preeclampsia," Journal of Chromatography B, vol. 753, no. 2, pp. 427-431, 2001.

[7] R. H. Glew, G. Melah, A. I. El-Nafaty, Y. Brandt, D. Morris, and D. J. VanderJagt, "Plasma and urinary free amino acid concentrations in preeclamptic women in northern Nigeria," Clinica Chimica Acta, vol. 342, no. 1-2, pp. 179-185, 2004.

[8] E. López-Quesada, M. A. Vilaseca, R. Artuch, E. Gómez, and J. M. Lailla, "Homocysteine and other plasma amino acids in preeclampsia and in pregnancies without complications," Clinical Biochemistry, vol. 36, no. 3, pp. 185-192, 2003.

[9] E. Turner, J. A. Brewster, N. A. B. Simpson, J. J. Walker, and J. Fisher, "Aromatic amino acid biomarkers of preeclampsia-a nuclear magnetic resonance investigation," Hypertension in Pregnancy, vol. 27, no. 3, pp. 225-235, 2008.

[10] E. Turner, J. A. Brewster, N. A. B. Simpson, J. J. Walker, and J. Fisher, "Plasma from women with preeclampsia has a low lipid and ketone body content-a nuclear magnetic resonance study," Hypertension in Pregnancy, vol. 26, no. 3, pp. 329-342, 2007.

[11] N. J. Robinson, L. J. Minchell, J. E. Myers, C. A. Hubel, and I. P. Crocker, "A potential role for free fatty acids in the pathogenesis of preeclampsia," Journal of Hypertension, vol. 27, no. 6, pp. 1293-1302, 2009.

[12] C. A. Hubel, F. Lyall, L. Weissfeld, R. E. Gandley, and J. M. Roberts, "Small low-density lipoproteins and vascular cell adhesion molecule-1 are increased in association with hyperlipidemia in preeclampsia," Metabolism, vol. 47, no. 10, pp. 1281-1288, 1998.

[13] G. A. Raouf, A. R. L. Al-Malki, N. Mansouri, and R. M. Mahmoudi, "Preliminary study in diagnosis and early prediction of preeclampsia by using FTIR spectroscopy technique," Life Science Journal, vol. 8, no. 2, pp. 453-464, 2011.

[14] G. J. Thomas Jr, "Raman spectroscopy of protein and nucleic acid assemblies," Annual Review of Biophysics and Biomolecular Structure, vol. 28, pp. 1-27, 1999.

[15] R. Tuma, "Raman spectroscopy of proteins: from peptides to large assemblies," Journal of Raman Spectroscopy, vol. 36, no. 4, pp. 307-319, 2005.

[16] G. J. Puppels, F. F. M. De Mul, C. Otto et al., "Studying single living cells and chromosomes by confocal Raman microspectroscopy," Nature, vol. 347, no. 6290, pp. 301-303, 1990.

[17] H. Deng, V. A. Bloomfield, J. M. Benevides, and G. J. Thomas, "Dependence of the Raman signature of genomic B-DNA on nucleotide base sequence," Biopolymers, vol. 50, no. 6, pp. 656666, 1999.

[18] M. Gniadecka, O. F. Nielsen, D. H. Christensen, and H. C. Wulf, "Structure of water, proteins, and lipids in intact human skin, hair, and nail," Journal of Investigative Dermatology, vol. 110, no. 4, pp. 393-398, 1998.

[19] B. Muik, B. Lendl, A. Molina-Díaz, and M. J. Ayora-Cañada, "Direct monitoring of lipid oxidation in edible oils by Fourier transform Raman spectroscopy," Chemistry and Physics of Lipids, vol. 134, no. 2, pp. 173-182, 2005.

[20] E. Zachariah, A. Bankapur, C. Santhosh, M. Valiathan, and D. Mathur, "Raman tweezers spectroscopy of live, single red and white blood cells," Journal of Photochemistry and Photobiology B, vol. 100, no. 3, pp. 113-116, 2010.

[21] A. C. de Luca, G. Rusciano, R. Ciancia et al., "Spectroscopical and mechanical characterization of normal and thalassemic red blood cells by Raman Tweezers," Optics Express, vol. 16, no. 11, pp. 7943-7957, 2008. 
[22] H. W. Han, X. L. Yan, R. X. Dong, G. Ban, and K. Li, "Analysis of serum from type II diabetes mellitus and diabetic complication using surface-enhanced Raman spectra (SERS)," Applied Physics B, vol. 94, no. 4, pp. 667-672, 2009.

[23] J. L. Pichardo-Molina, C. Frausto-Reyes, O. Barbosa-García et al., "Raman spectroscopy and multivariate analysis of serum samples from breast cancer patients," Lasers in Medical Science, vol. 22, no. 4, pp. 229-236, 2007.

[24] T. W. Koo, A. J. Berger, I. Itzkan, G. Horowitz, and M. S. Feld, "Reagentless blood analysis by near-infrared Raman spectroscopy," Diabetes Technology \& Therapeutics, vol. 1, no. 2, pp. 153157, 1999.

[25] J. Y. Qu, B. C. Wilson, and D. Suria, "Concentration measurements of multiple analytes in human sera by near-infrared laser Raman spectroscopy," Applied Optics, vol. 38, no. 25, pp. 5491-5498, 1999.

[26] A. M. K. Enejder, T. W. Koo, J. Oh et al., "Blood analysis by Raman spectroscopy," Optics Letters, vol. 27, no. 22, pp. 2004-2006, 2002.

[27] A. J. Berger, T. W. Koo, I. Itzkan, G. Horowitz, and M. S. Feld, "Multicomponent blood analysis by near-infrared Raman spectroscopy," Applied Optics, vol. 38, no. 13, pp. 2916-2926, 1999.

[28] D. Qi and A. J. Berger, "Chemical concentration measurement in blood serum and urine samples using liquid-core optical fiber Raman spectroscopy," Applied Optics, vol. 46, no. 10, pp. 17261734, 2007.

[29] C. M. Krishna, N. B. Prathima, R. Malini et al., "Raman spectroscopy studies for diagnosis of cancers in human uterine cervix," Vibrational Spectroscopy, vol. 41, no. 1, pp. 136-141, 2006.

[30] E. B. Hanlon, R. Manoharan, T. W. Koo et al., "Prospects for in vivo Raman spectroscopy," Physics in Medicine and Biology, vol. 45, no. 2, pp. R1-R59, 2000.

[31] K. Das, N. Stone, C. Kendall, C. Fowler, and J. Christie-Brown, "Raman spectroscopy of parathyroid tissue pathology," Lasers in Medical Science, vol. 21, no. 4, pp. 192-197, 2006.

[32] H. Wills, R. Kast, C. Stewart et al., "Raman spectroscopy detects and distinguishes neuroblastoma and related tissues in fresh and (banked) frozen specimens," Journal of Pediatric Surgery, vol. 44, no. 2, pp. 386-391, 2009.

[33] B. R. Wood, L. Hammer, and D. McNaughton, "Resonance Raman spectroscopy provides evidence of heme ordering within the functional erythrocyte," Vibrational Spectroscopy, vol. 38, no. 1-2, pp. 71-78, 2005.

[34] G. T. Webster, L. Tilley, S. Deed, D. McNaughton, and B. R. Wood, "Resonance Raman spectroscopy can detect structural changes in haemozoin (malaria pigment) following incubation with chloroquine in infected erythrocytes," FEBS Letters, vol. 582, no. 7, pp. 1087-1092, 2008.

[35] J. R. Ferraro, K. Nakamoto, and C. W. Brown, Raman Spectroscopy, Elsevier, New York, NY, USA, 2nd edition, 2003.

[36] D. Rohleder, W. Kiefer, and W. Petrich, "Quantitative analysis of serum and serum ultrafiltrate by means of Raman spectroscopy," Analyst, vol. 129, no. 10, pp. 906-911, 2004.

[37] Y. Shi and L. Wang, "Collective vibrational spectra of $\alpha$ - And $\gamma$-glycine studied by terahertz and Raman spectroscopy," Journal of Physics D, vol. 38, no. 19, pp. 3741-3745, 2005.

[38] T. Miura, H. Takeuchi, and I. Harada, "Raman spectroscopic characterization of tryptophan side chains in lysozyme bound to inhibitors: role of the hydrophobic box in the enzymic function," Biochemistry, vol. 30, no. 24, pp. 141-157, 1991.

[39] N. Stone, C. Kendall, J. Smith, P. Crow, and H. Barr, "Raman spectroscopy for identification of epithelial cancers," Faraday Discussions, vol. 126, pp. 141-157, 2004.

[40] Y. S. Mary, L. Ushakumari, B. Harikumar, H. T. Varghese, and C. Y. Panickerb, "FT-IR, FT-Raman and SERS spectra of L-proline," Journal of the Iranian Chemical Society, vol. 6, no. 1, pp. 138$144,2009$. 
[41] K. L. Aubrey and G. J. Thomas Jr, "Raman spectroscopy of filamentous bacteriophage Ff (fd, M13, f1) incorporating specifically-deuterated alanine and tryptophan side chains: assignments and structural interpretation," Biophysical Journal, vol. 60, no. 6, pp. 1337-1349, 1991.

[42] Z. Huang, A. McWilliams, H. Lui, D. I. McLean, S. Lam, and H. Zeng, "Near-infrared Raman spectroscopy for optical diagnosis of lung cancer," International Journal of Cancer, vol. 107, no. 6, pp. 1047-1052, 2003.

[43] Y. K. Sze, A. R. Davis, and G. A. Neville, "Raman and infrared studies of complexes of mercury(II) with cysteine, cysteine methyl ester, and methionine," Inorganic Chemistry, vol. 14, no. 8, pp. 1969-1974, 1975.

[44] G. Zhu, X. Zhu, Q. Fan, and X. Wan, "Raman spectra of amino acids and their aqueous solutions," Spectrochimica Acta A, vol. 78, no. 3, pp. 1187-1195, 2011.

[45] J. W. Chan, D. Motton, J. C. Rutledge, N. L. Keim, and T. Huser, "Raman spectroscopic analysis of biochemical changes in individual triglyceride-rich lipoproteins in the pre- and postprandial state," Analytical Chemistry, vol. 77, no. 18, pp. 5870-5876, 2005.

[46] E. B. Hanlon, R. Manoharan, T. W. Koo et al., "Prospects for in vivo Raman spectroscopy," Physics in Medicine and Biology, vol. 45, no. 2, pp. R1-R59, 2000.

[47] J. J. Baraga, M. S. Feld, and R. P. Rava, "In situ optical histochemistry of human artery using near infrared Fourier transform Raman spectroscopy," Proceedings of the National Academy of Sciences of the United States of America, vol. 89, no. 8, pp. 3473-3477, 1992.

[48] R. G. Brereton, Applied Chemometrics for Scientists, John Wiley \& Sons, Chichester, UK, 2007.

[49] J. E. Jackson, A User Guide to Principal Component Analysis, John Wiley \& Sons, New York, NY, USA, 1991.

[50] A. R. Webb, Statistical Pattern Recognition, John Wiley \& Sons, Chichester, UK, 2nd edition, 2002.

[51] S. P. Wolff, A. Garner, and R. T. Dean, "Free radicals, lipids and protein degradation," Trends in Biochemical Sciences, vol. 11, no. 1, pp. 27-31, 1986.

[52] B. S. Berlett and E. R. Stadtman, "Protein oxidation in aging, disease, and oxidative stress," Journal of Biological Chemistry, vol. 272, no. 33, pp. 20313-20316, 1997. 


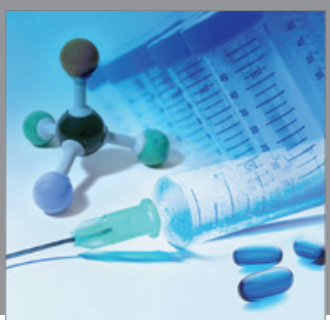

International Journal of

Medicinal Chemistry

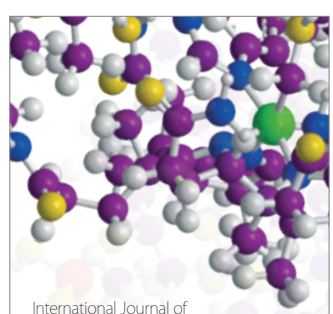

Carbohydrate Chemistry

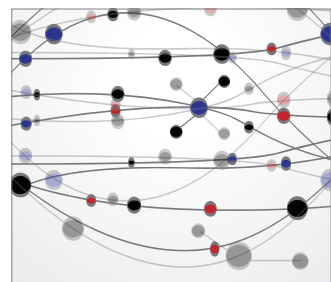

The Scientific World Journal
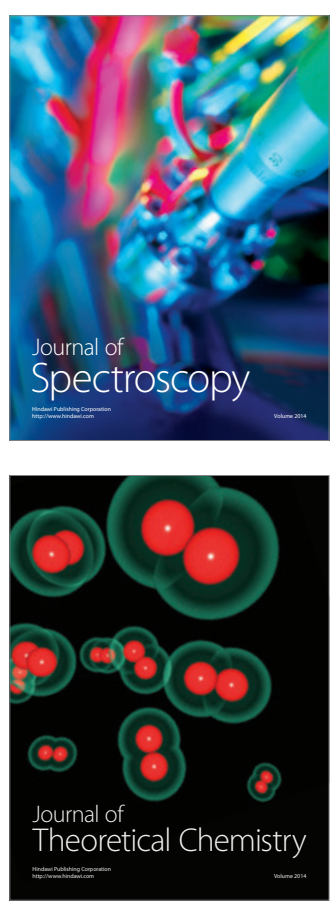
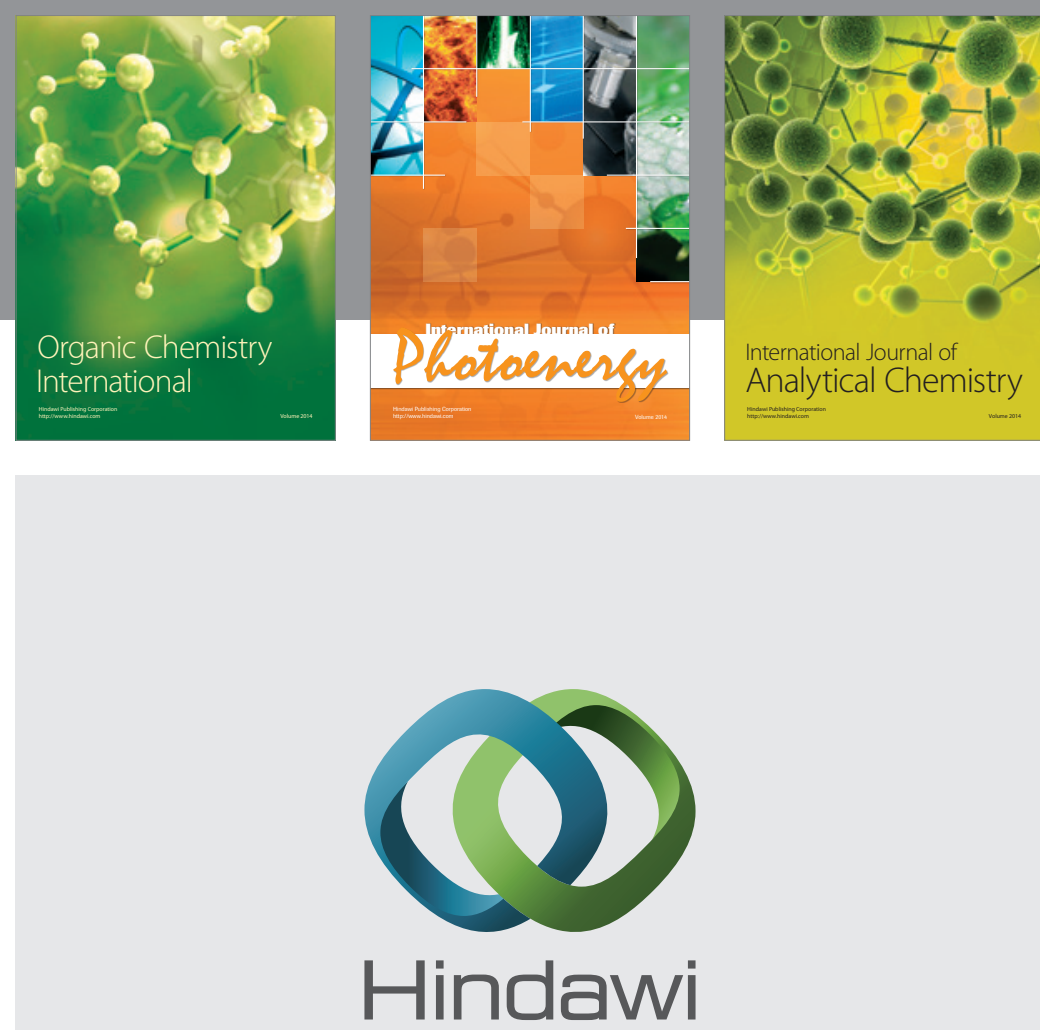

Submit your manuscripts at

http://www.hindawi.com
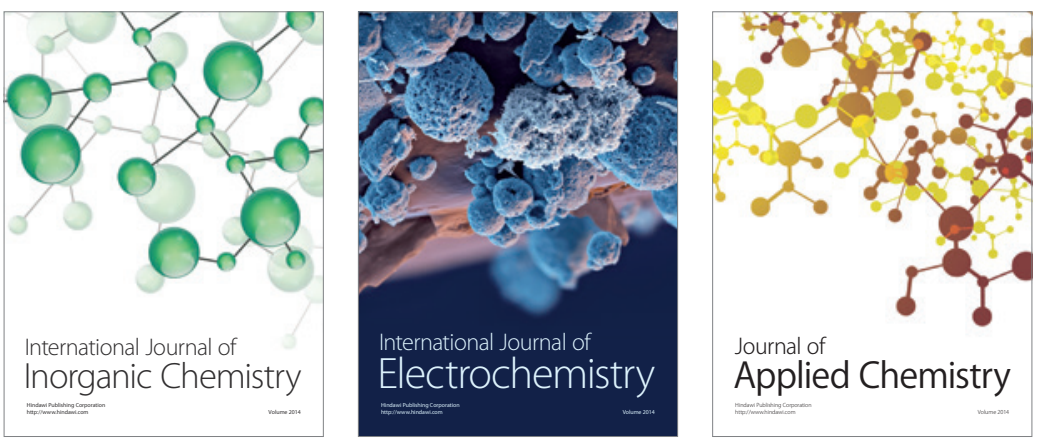

Journal of

Applied Chemistry
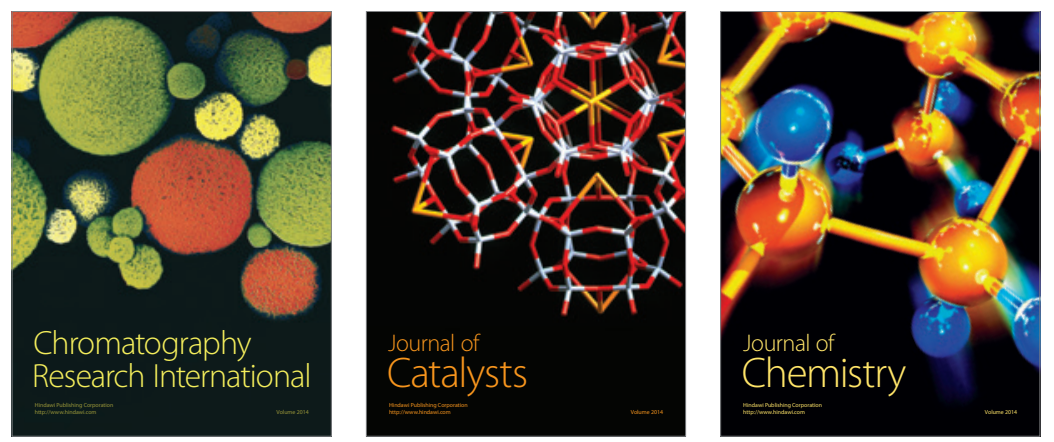
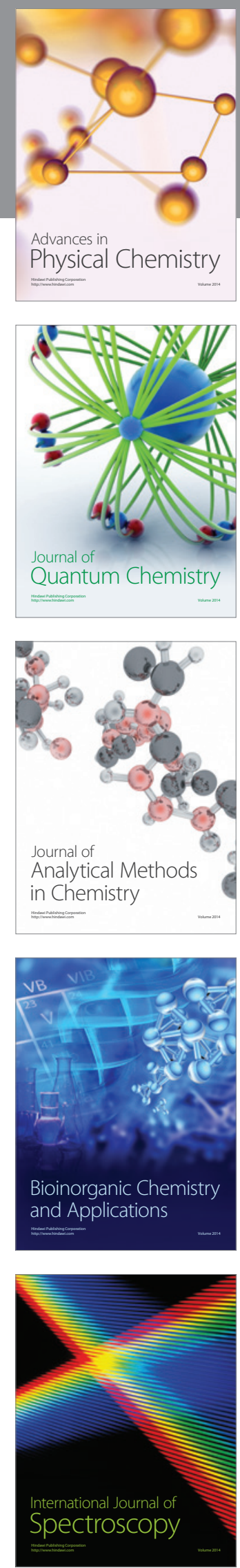